\title{
IMPLEMENTATION OF A SURFACE BASED COUPLING APPROACH IN A HIGH- ORDER DG AEROACOUSTICS PROPAGATION SOLVER
}

M. Muriel Gracia, W. De Roeck and W. Desmet

\section{ABSTRACT:}

A surface based coupling approach similar to Curle's acoustic analogy has been implemented. This method allows for the computation of aerodynamically generated noise propagation accounting for scattering effects produced by surface boundaries. For low Mach number problems with acoustically compact sources of sound, the advantages of choosing a surface based approach to use unsteady sources for acoustics propagation are twofold. On one hand, the computational domain used during CFD simulations can be significantly reduced, and the data for the sources has to be stored uniquely in the noise generating surfaces. On the other hand, no truncation on the sources information is necessary. In this work, the sound propagation produced by the lift and drag forces acting on a tandem cylinders configuration is calculated using a discontinuous Galerkin method to solve the linearized Euler equations. The proposed coupling approach is first verified for the acoustics propagation of the sound generated by the lift and drag dipoles originated by flow passing a single cylinder, where no scattering occurs. After verification, the methodology is applied to the tandem cylinders problem and the scattering effects are investigated. 